Bibliotecas. Vol 36, N¹ Especial, 2018. EISSN: 1659-3286

URL: http://www.revistas.una.ac.cr/index.php/bibliotecas/index

DOI: http://dx.doi.org/10.15359/rb.36-1.6

Licencia: Creative Commons (BY-NC-ND) 4.0 Internacional

\title{
Perfil del docente de la Facultad de Ciencias de la Información de la Universidad Autónoma de San Luis Potosí
}

\section{Profile of the Teachers of the Faculty of Sciences of the Information of the Universidad Autónoma de San Luis Potosí}

Dra. Rosa María Martínez Rider* Universidad Autónoma de San Luis Potosí, México Dra. Adriana Mata Puente** Universidad Autónoma de San Luis Potosí, México Dr. Eduardo Oliva Cruz ${ }^{* \star \star}$ Universidad Autónoma de San Luis Potosí, México

\section{Resumen}

Se presentan los resultados de las características del perfil docente del bibliotecólogo en la Facultad de Ciencias de la Universidad Autónoma de San Luis Potosí, como parte de una investigación Iberoamericana del Seminario de Educación Bibliotecológica, coordinado por la Dra. Lina Escalona Ríos en el Instituto de Investigaciones bibliotecológicas y de Información de la UNAM.

El objetivo fue analizar la congruencia entre el perfil del docente y el modelo educativo que permea el currículum de Gestión de Información de la Facultad de Ciencias de la Información (FCl) de la Universidad Autónoma de San Luis Potosí (UASLP), México.

Los resultados de la encuesta de tipo exploratoria aplicada a los docentes fueron representativos para los profesores asignatura, quienes respondieron la mayor parte de las encuestas.

Palabras clave: Educación Bibliotecológica; Perfil docente; DocenteBibliotecología. 


\begin{abstract}
The results of the characteristics of the teaching profile of the librarian at the Facultad de Ciencias de la Información of the Universidad Autónoma de San Luis Potosí are presented as part of an Ibero-American research project of the Library Education Seminar, coordinated by Phd. Lina Escalona Ríos at the Instituto de de Investigaciones Bibliotecológicas y de Información of the UNAM. The objective was to analyze the congruence between the teacher profile and the educational model that permeates the Information Management curriculum of the Facultad de Ciencias de la Información (FCl) of the Universidad Autónoma de San Luis Potosí (UASLP), México.
\end{abstract}

Key words: Library Education; Teaching profile; Teacher-Librarianship.

\title{
I. Introducción
}

El perfil ideal del docente presenta un sinnúmero de características deseables que pueden ser superadas o alcanzables. No obstante, es fundamental identificar los rasgos de la planta de profesores en cualquier institución educativa para tomar acciones que contribuyan a la calidad y la mejora continua, mediante el análisis de las fortalezas o debilidades que se presentan. Asimismo, las concepciones sobre el perfil del profesor y el modelo educativo deben ser congruentes con la realidad, considerando la parte teórica y práctica del currículum vivido.

\section{El Perfil docente}

El perfil académico se estructura a través de un conjunto de competencias para la realización del trabajo de los profesores que involucra, además de la enseñanza, una serie de actividades colegiadas y de investigación sobre la práctica docente, la cual se transforma ante los nuevos escenarios de la información, del avance del conocimiento, la innovación, los cambios 
sociales y la política educativa. Por ejemplo, el conocimiento de la biología, la física y, en general, de toda la ciencia en su faceta dura o social avanza vertiginosamente, donde la información juega un papel primordial en su desarrollo y el bibliotecólogo especialista en la organización, difusión y conservación es quien la proporciona para satisfacer los requerimientos de educación o recreación de las personas. Cualquier docente debe adecuarse con estos cambios para formar a los nuevos profesionales de la información y que estos contribuyan a formar ciudadanos responsables, críticos y propositivos con miras a mejorar la toma de decisiones, la calidad de vida o el progreso del conocimiento.

El perfil docente se caracteriza por ser dinámico; por renovar las estrategias de aprendizaje acordes con el contexto actual; por llevar la realidad al aula; y por fomentar la participación activa de los estudiantes, además de los fundamentos disciplinares y pedagógicos para atender el qué, el cómo y el para qué. Bozu y Canto (2009) acotan un aspecto imprescindible sobre el profesorado "el devenir histórico, marcado por el modelo educativo, institucional, legislativo y social del docente" (p.90) en función de la ubicación geográfica, el idioma, la cultura, la cosmovisión, los avances en las ramas del saber y la tecnología, entre otros factores.

Díaz Barriga (2005) indica que "es frente a esta multiplicidad que cada docente tiene que construir su estilo didáctico y desarrollar estrategias de enseñanza" (párr. 4), considerando la finalidad de la educación. En este orden de ideas, Bozu y Canto (2009) destacan el papel de la metodología didáctica que se elige en función de la especificidad del trabajo a realizar en cada momento y en cada escenario; el uso de los recursos didácticos adecuados al desarrollo de la asignatura; la incorporación indispensable de las nuevas tecnologías de la información y comunicación en la docencia; la atención a las estrategias que emplean los alumnos para asimilar la información para transferirla en la práctica o en otros contextos similares; y la escucha activa a los estudiantes para identificar sus problemas y sus dificultades. 
Los procesos de enseñanza y aprendizaje son holísticos y varían según el tamaño y características de los grupos de estudiantes, donde el estudiante se apropia del conocimiento, desarrolla nuevas habilidades y destrezas, reafirma sus valores y su posición frente a la complejidad de la disciplina o profesión. Álvarez (2011) señala que se "requiere de profundos cambios, de nuevos perfiles tanto de estudiantes como de docentes" (p.11) en el Siglo XXI y que el profesor debe "comprometerse, mediante la reflexión constante sobre su tarea docente, en espacios de construcción individual y grupal, y con aportes significativos, de forma tal que pueda enfrentarse con los numerosos dilemas y retos que se presentan en este milenio" ( $p$. 101). Otras características definen el perfil docente, no solo sus competencias o estrategias didácticas, las cuales inciden en el modelo educativo, debido a ello a continuación se presentan las generalidades del programa educativo del que se desprendieron los resultados de esta investigación.

\section{El Plan de estudios de Gestión de Información de la Facultad de Ciencias de la Información de la UASLP}

El modelo educativo, según Tünnermann (2008), es "La concreción, en términos pedagógicos, de los paradigmas educativos que una institución profesa y que sirve de referencia para todas las funciones que cumple" (p. 15). El autor incorpora una posición epistemológica, educativa y social que confluye en la formación de profesionales en diferentes niveles y áreas del saber.

Un modelo educativo se compone de varias dimensiones externas (Figura 1) que se consideran en el proyecto curricular (Figura 2) que se establece en cualquier oferta educativa porque este reproduce la cultura, entendida como la ciencia, las normas sociales de convivencia, entre otros aspectos (Gimeno Sacristán, 1989). 


\section{Figura 1}

Dimensiones externas de un modelo educativo

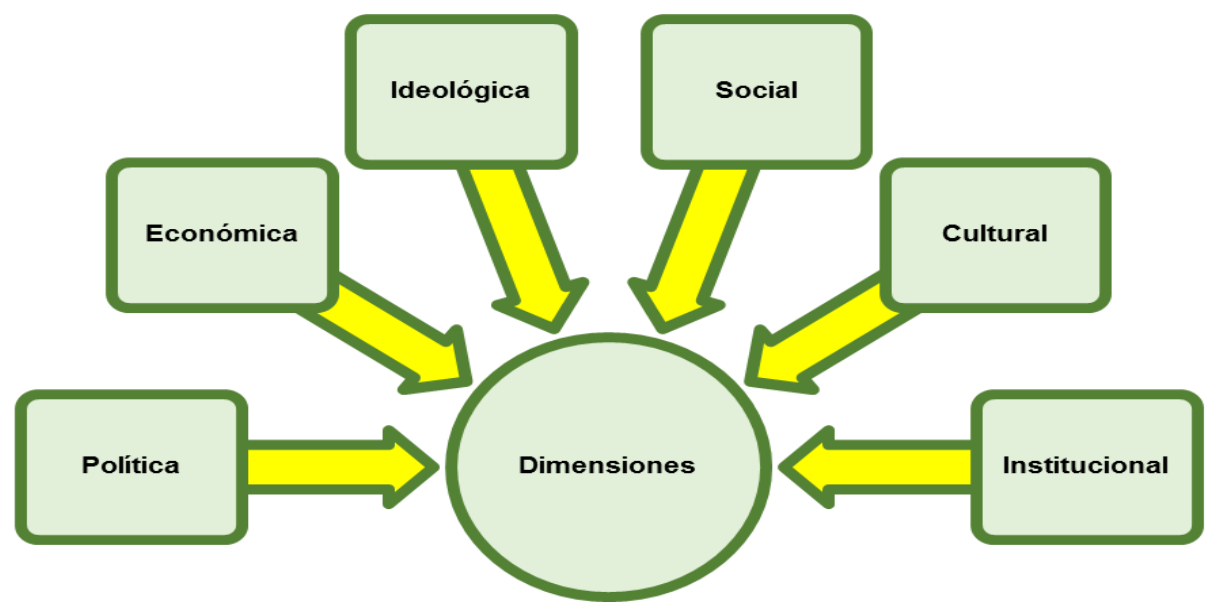

Fuente: Elaboración propia de los autores.

\section{Figura 2}

\section{Dimensiones del proyecto educativo}

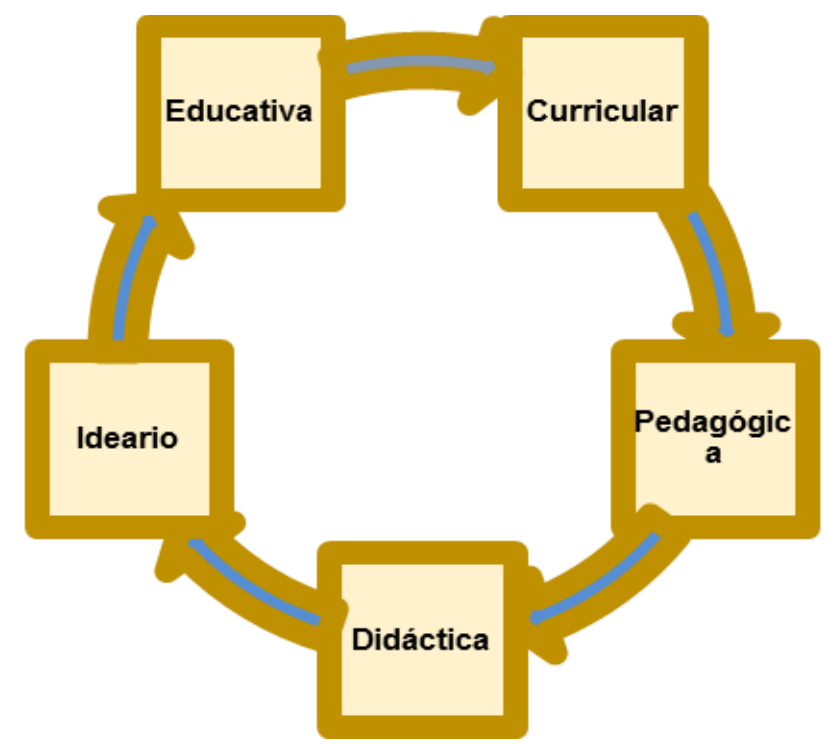

Fuente: Elaboración propia de los autores.

La Facultad de Ciencias de la Información se creó como Licenciatura en Biblioteconomía en 1984, posteriormente se elevó a rango de Escuela de Biblioteconomía en 1997. En 1998 se designa como Escuela de Bibliotecología e Información y en 2006 el H. Consejo Directivo 
Universitario aprueba el nombre de Escuela de Ciencias de la Información, al incorporar la carrera de Archivología en ese mismo año.

Las licenciaturas ofertadas hasta el 2014 fueron Biblioteconomía en sus inicios o Bibliotecología después de las modificaciones en el nombre y Archivología, las cuales cerraron para ofertar nuevas carreras: Gestión de la Información (Gl) y Gestión Documental y Archivística (GDA). Sin embargo, todos los profesores de la especialidad que imparten cátedra en Gestión de Información tienen título en Biblioteconomía o Bibliotecología. Hasta 2018 egresa la primera generación de estas nuevas licenciaturas.

La posición epistemológica del proyecto curricular es la ciencia bibliotecológica, a partir del objeto de estudio definido como Información; la educativa es el diseño por competencias; y el social atender las necesidades de información de los diferentes sectores sociales. El Plan de estudios de la carrera de Gestión de Información (2014) define el perfil profesional de los egresados como "El gestor de la información debe ser competente en la administración de recursos informativos, humanos, financieros y tecnológicos. Visionario para predecir la demanda de futuros servicios y proactivo en su desarrollo dentro de un contexto de política de información" (p.9)

Los objetivos son:

a) Formar profesionales en el manejo de la información documental para la planeación, evaluación y toma de decisiones en los ámbitos organizacional, docente y de investigación.

b) Desarrollar en el estudiante conocimientos, habilidades y actitudes con un sentido crítico, creativo, libre y ético para inducir, generar y adaptarse a los cambios que demanda la sociedad.

c) Buscar la excelencia, innovación y la mejora continua a través de una constante actualización y espíritu de superación, conservando los valores que promueve la educación superior. 
El plan se divide en tres grandes áreas:

1. Básica: que es de tronco común.

2. Profesional: de la especialidad de cada carrera.

3. De profundización: optativas.

Y consta de los siguientes módulos curriculares:

a. Servicios (11 materias)

b. Administración (13 materias)

c. TIC (7 materias)

d. Organización (11 materias)

e. Patrimonio (4 materias)

f. Normalización (7 materias)

Con un total de 53 asignaturas, más las materias obligatorias de inglés, el Servicios Social y las Prácticas Profesionales, los profesores de tiempo completo deben cumplir con las funciones de docencia, investigación, gestión (Academias, Comisión Curricular, Planeación, etc.), tutoría y asesoría a los estudiantes. Los profesores asignatura varían de una a ocho horas diarias frente a grupo. Como este plan de estudios es nuevo, se reasignan las materias entre los profesores asignatura y se reorganizan entre los de tiempo completo.

\section{Metodología}

El planteamiento del problema que originó este trabajo fue ¿Cuáles son las características del docente de la $\mathrm{FCl}$ en cuanto a su formación, experiencia docente, práctica docente, área de investigación y educación continua? Para ello se aplicó una parte de la encuesta derivada de los trabajos del Seminario mencionado que respondieron cinco profesores de tiempo completo y 15 profesores de tiempo parcial, es decir 20 docentes de un total de 29 profesores. En virtud 
del cambio curricular, de las adaptaciones que conlleva y la movilidad de los profesores asignatura, solo se aplicaron 14 preguntas del total determinado en el Seminario, que se relacionan con datos generales, docencia, investigación, experiencia y educación continua.

Se realizó este trabajo en docentes con especialidad en Bibliotecología que impartieron clases en Gl, durante el ciclo enero-junio de 2017 de un total de 29. El instrumento que fue diseñado y piloteado por el grupo de investigación del Seminario de Educación Bibliotecológica, contiene preguntas relacionadas con los siguientes puntos:

1. Sexo

2. Edad

3. Nivel de docencia

4. Situación actual como profesor

5. Antigüedad

6. Formación académica

7. Experiencia docente

8. Cursos de actualización

9. Cinco últimos cursos

10. Área a la que corresponden los cursos

11. Asignaturas impartidas

12. Área de las asignaturas

13. Cursos de formación pedagógica

14. Cinco últimos cursos

15. Uso de TIC
16. Plataformas y redes usadas

17. Investigación

18. Área de investigación

19. Tres productos de investigación

20. Tipo de institución laboral

21. Área de ejercicio laboral

22. Tiempo de experiencia laboral

23. Área laboral con actividad docente

24. Cursos de capacitación o actualización laboral

25. Últimos cinco cursos

26. Participación en eventos

27. Tres últimos eventos

28. Talleres impartidos

29. Tres últimos cursos

El que fue aplicado en la $\mathrm{FCl}$ incluyó los siguientes datos:
1. Sexo
3. Antigüedad
2. Edad
4. Nivel de estudios 
5. Experiencia docente

6. Cursos de actualización

7. Cinco últimos cursos

8. Uso de TIC

9. Investigación
10.Tipo de institución laboral

11. Área de ejercicio laboral

12.Área laboral con actividad docente

13. Talleres impartidos

14. Tres últimos cursos

A continuación se presentan las respuestas obtenidas en el estudio realizado a la planta docente del Plan de Estudio de la licenciatura en Gestión de la Información.

\section{Resultados}

Del total de la planta docente se tiene un porcentaje del $55 \%$ de mujeres, en donde el resto, $45 \%$ corresponde a los hombres, cuya preponderancia se da en lo referente al género femenino, cabe señalar que esta tendencia se ha dado a lo largo de la historia de la Facultad hasta el momento de este estudio (Figura 1)

Figura 3

Docentes por género de la carrera de Gestión de la Información

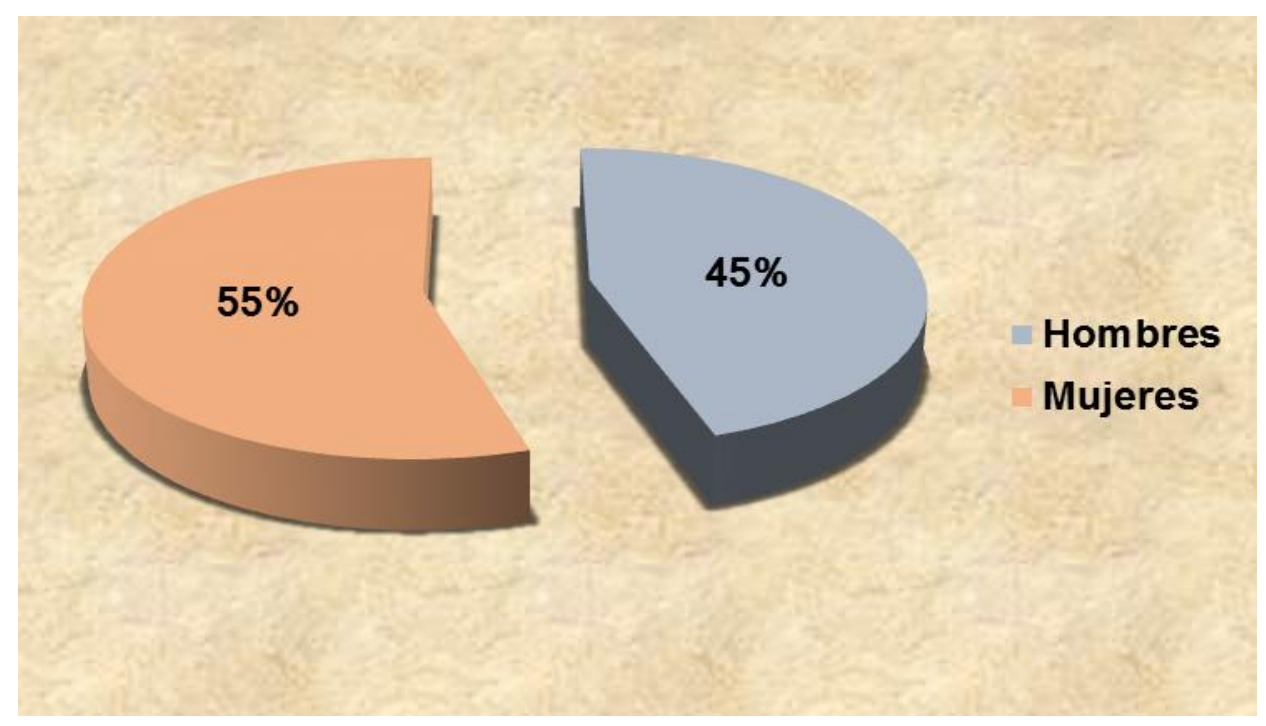

Fuente: Elaboración propia de los autores. 
En relación con la edad biológica por cada uno de los docentes que imparten cursos en el plan de estudios de Gestión de la Información, se tiene que el grupo ubicado entre los 40 y 45 años representa el porcentaje más alto con un $25 \%$; le sigue el grupo de docentes ubicados en el rango de los 31 a 35 años con porcentaje de $20 \%$; los rangos que van de los 25 a 30 y de los 36 a 40 años representan un 15\% respectivamente; quedando el rango ubicado entre los 46 a 50 años con un porcentaje del 10\% (Figura 4).

Figura 4

Rangos de edad en años de los docentes de la licenciatura en Gestión de la Información

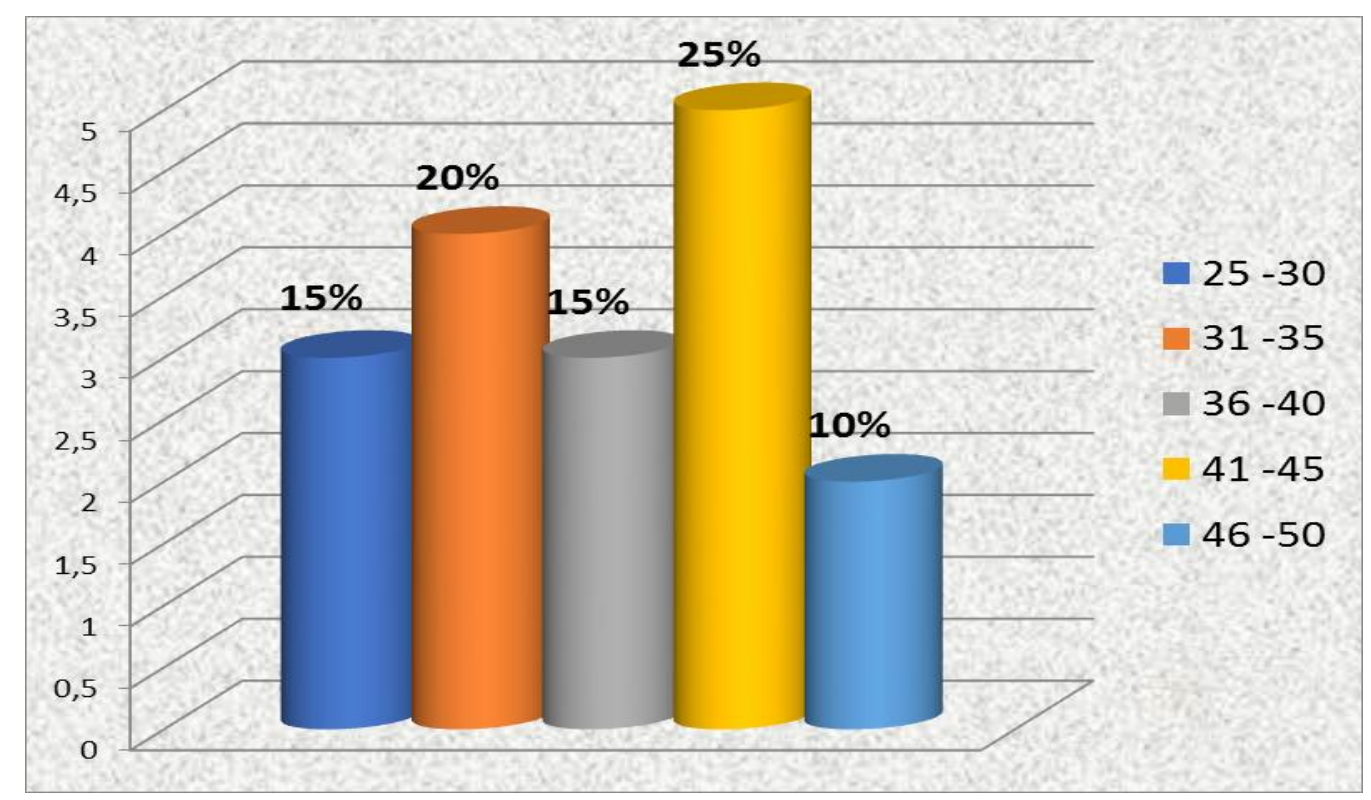

Fuente: Elaboración propia de los autores.

En lo referente a los grados académicos de los docentes del programa en Gestión de la Información, se obtuvieron los siguientes resultados: la planta está integrada por una mayoría de profesores con el grado de licenciatura y maestría con un total del $45 \%$; le siguen aquellos docentes que cuentan solamente con el grado de licenciatura; para continuar con los que tienen maestría y doctorado con un $10 \%$; y, por último, están los docentes que tiene diplomado en el área y maestría con un 5\% (Figura 5). 


\section{Figura 5}

\section{Nivel de estudios de los docentes en GI}

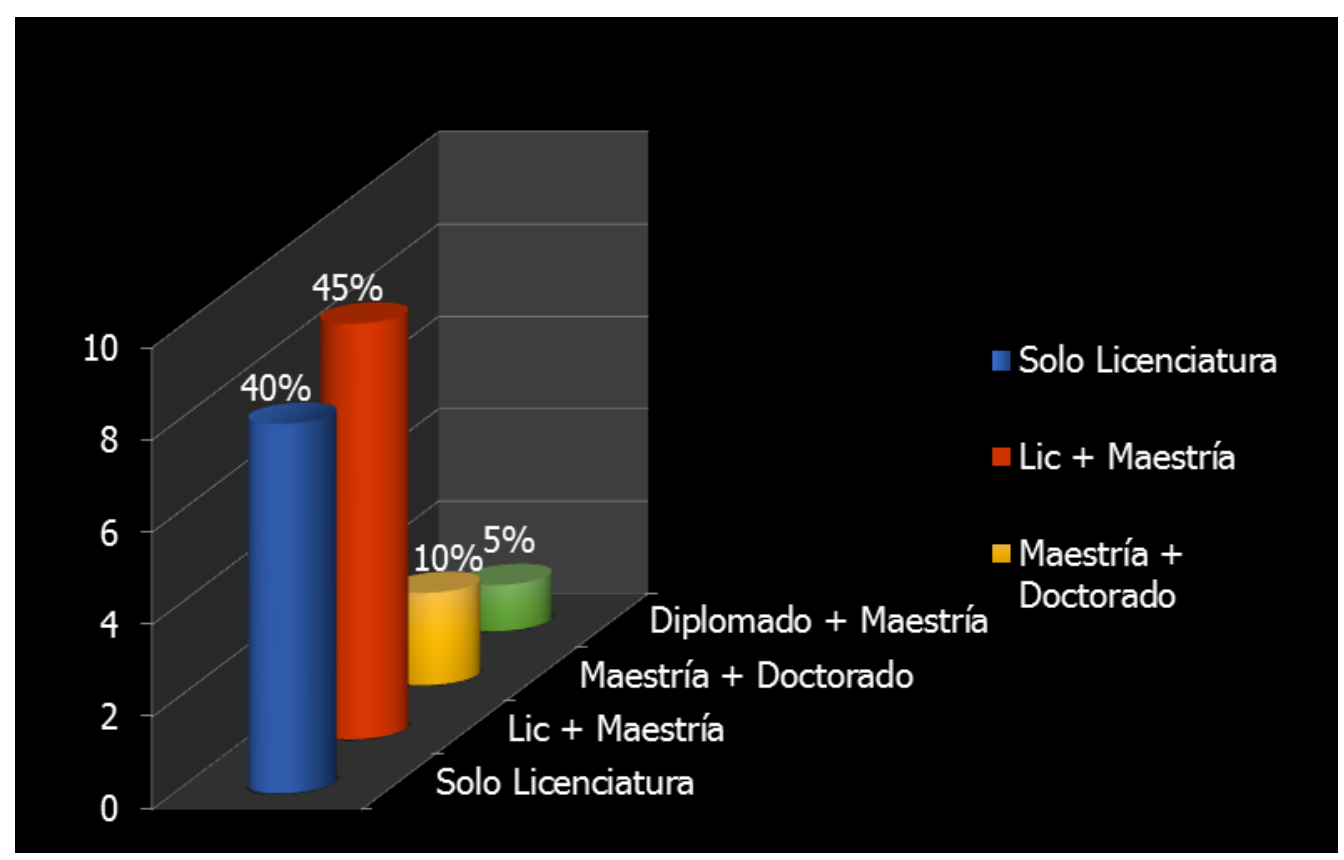

Fuente: Elaboración propia de los autores.

Respecto de la experiencia docente de la planta de profesores de la licenciatura en Gestión de la Información, la encuesta arrojó los siguientes resultados: el 35\% afirmó contar con una experiencia no mayor de 5 años en el ejercicio docente; le siguieron con un porcentaje del $30 \%$ los docentes que cuentan con experiencia entre un rango de 6 a 10 años; con un 20\% de experiencia se encuentran los docentes que tienen experiencia entre los 11 y 15 años; le siguen con un $10 \%$ aquellos docentes que tienen experiencia de 26 o más años; y por último en un rango de 21 a 25 años se encuentra un 5\% de los profesores de la planta docente de este programa (Figura 6). 


\section{Figura 6}

\section{Experiencia docente en años}

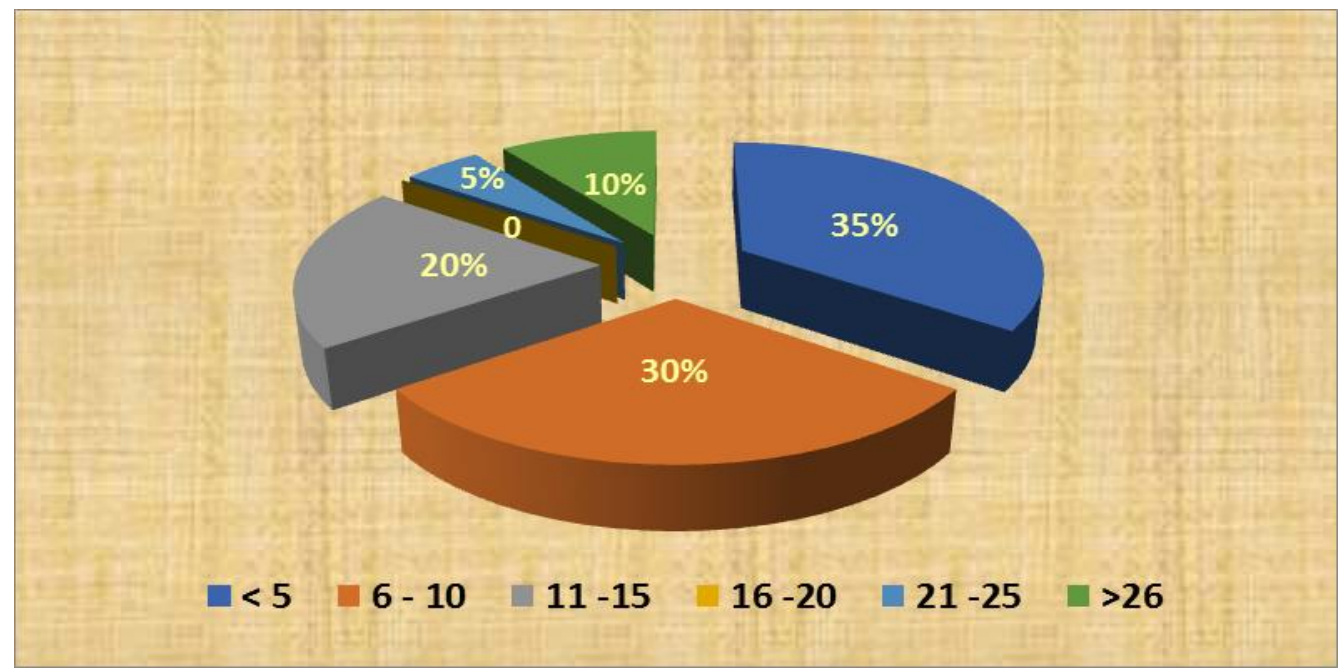

Fuente: Elaboración propia de los autores.

En el tema de la actualización disciplinar los docentes externaron que no se capacita un $20 \%$, mientras que el resto $80 \%$ sí se actualiza en su campo disciplinar (Figura 6).

\section{Figura 6}

\section{Cursos de actualización disciplinar}

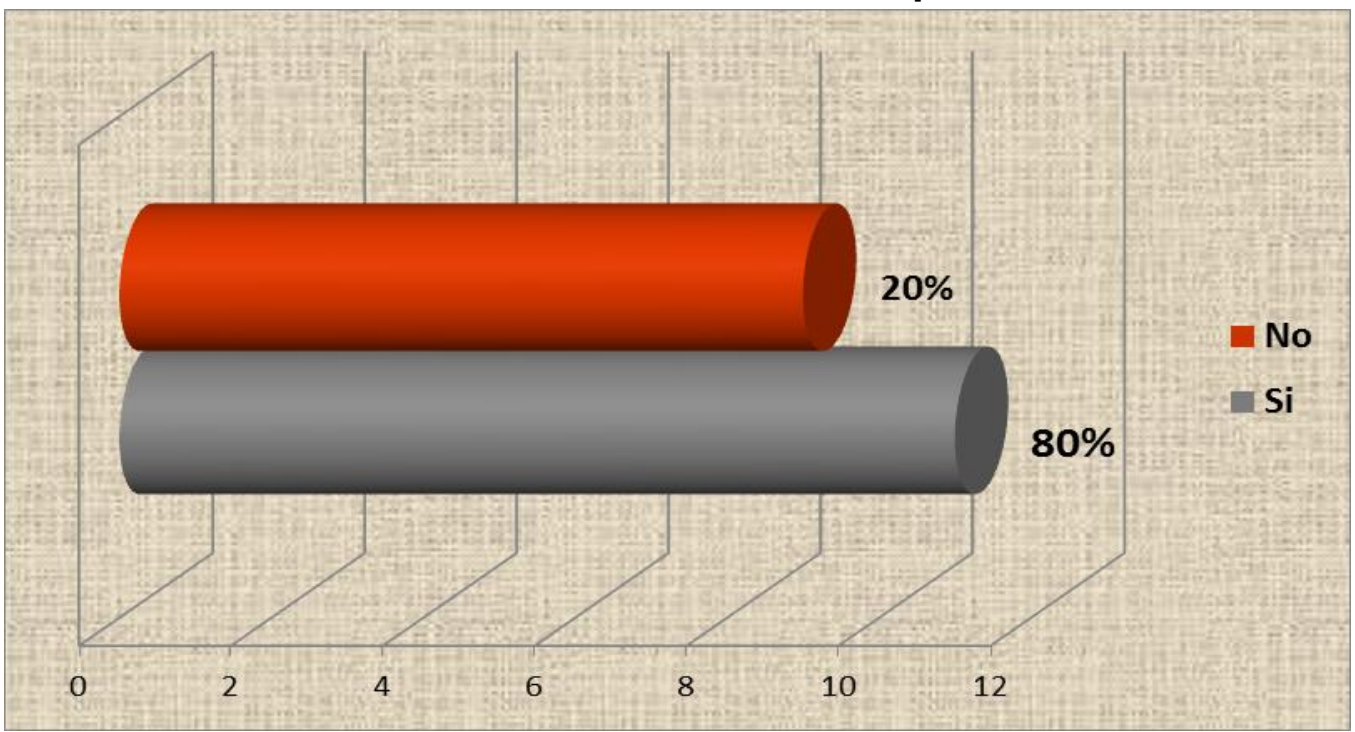

Fuente: Elaboración propia de los autores. 
Las herramientas de tecnologías de información y comunicación (TIC) apoyan las actividades docentes, los profesores manifestaron en un $5 \%$ que usan la plataforma Chamillo ${ }^{\odot}$, la cual sirve como apoyo de aula virtual; le sigue el uso del presentador de imágenes denominado Prezi@ con un 26\%; en lo que se refiere a la búsqueda de datos, así como el apoyo en video un $13 \%$ de profesores utilizan estas herramientas; solo el $8 \%$ de los docentes utilizan el Facebook; el recurso más utilizado como apoyo para la docencia es el presentador de imágenes Power Point ${ }^{\odot}$ con un $38 \%$; y $5 \%$ se auxilia con las herramientas de Internet, como las infografías (Figura 7).

Figura 7

Recursos con TIC utilizados en la docencia

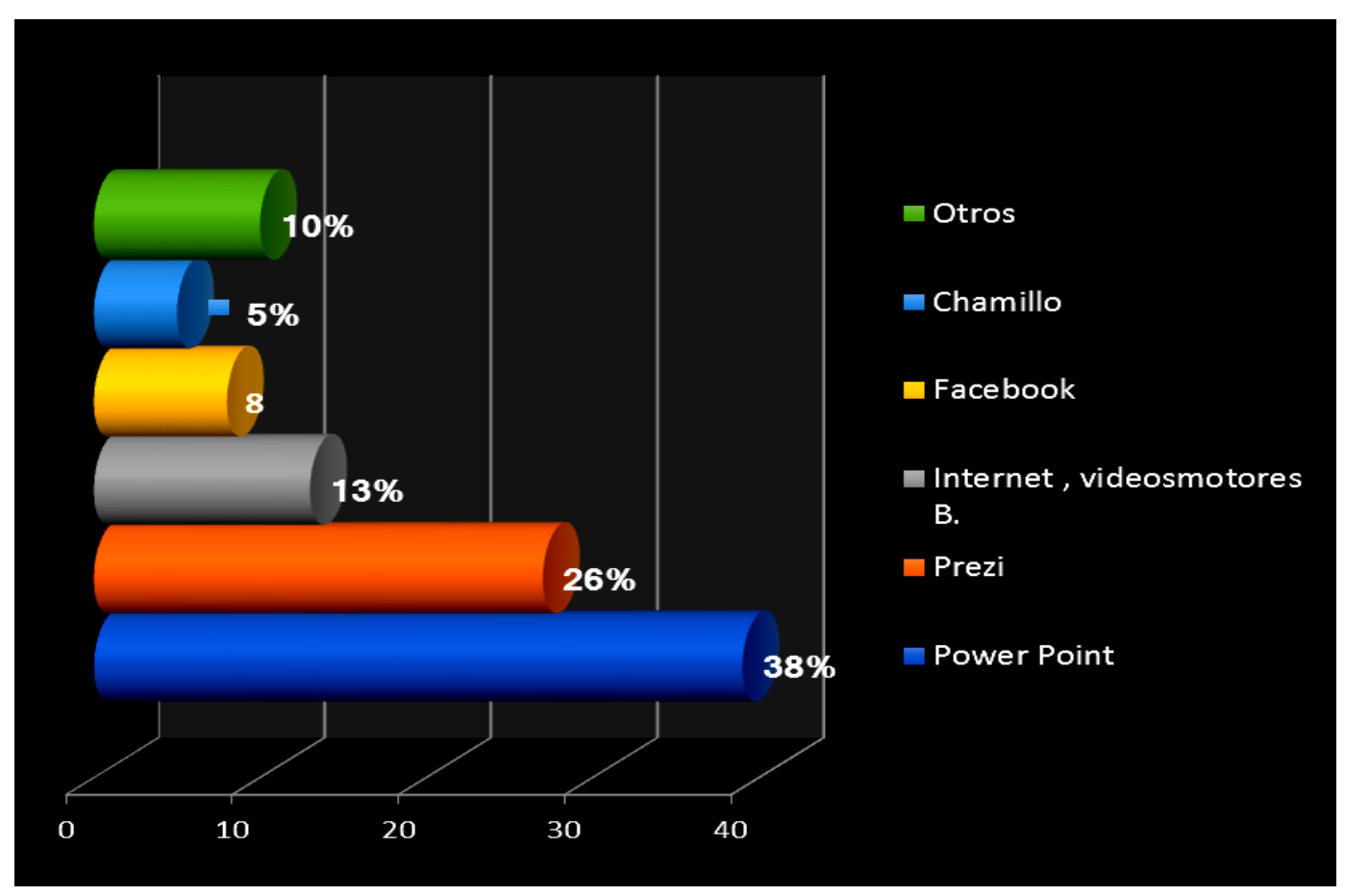

Fuente: Elaboración propia de los autores.

En la investigación, como parte de la función de los docentes, en el programa de Gestión de la Información, se obtuvieron los siguientes datos: el 30\% de la planta docente manifestó que sí es una actividad inherente a su actividad docente; el $45 \%$ manifestó que no; y el $25 \%$ dijo que no formaba parte de su quehacer como docentes (Figura 8). 


\section{Figura 8}

\section{Investigación como parte de la labor docente}

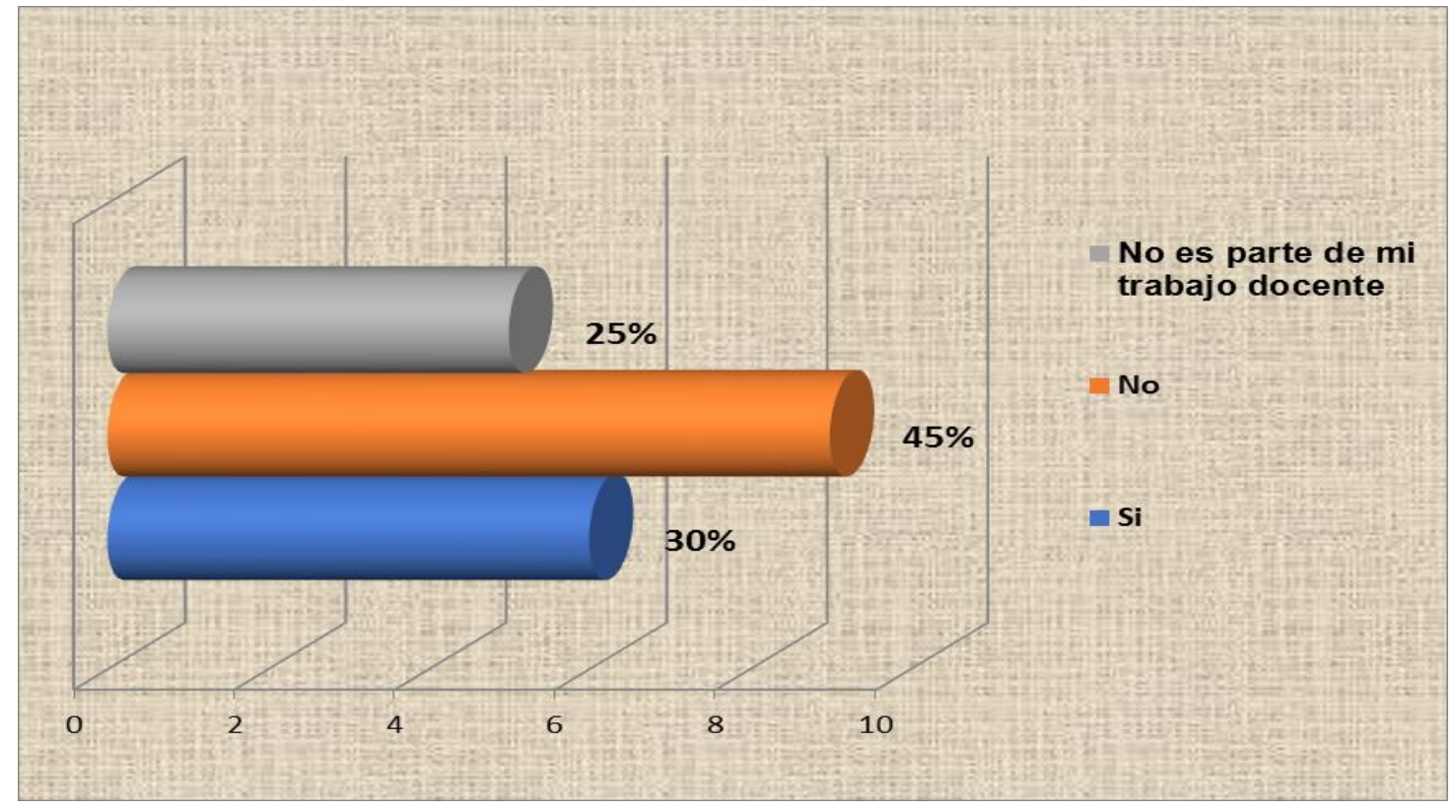

Fuente: Elaboración propia de los autores.

Sobre los centros de información o unidad de información donde se desempeñan los profesores asignatura de Gestión de la Información se obtuvieron los siguientes datos: el $54.5 \%$ se desempeña en bibliotecas universitarias; el 18.2\% en centros de documentación y de información; en igual porcentaje el $9.1 \%$ se desempeña en bibliotecas escolares, archivos u otro no especificado (Figura 9).

Sobre el desempeño profesional los docentes asignatura manifestaron en un $35 \%$ que se dedican a la organización de la información; el 30\% a prestar servicios de información; el 25\% a la administración de la información; y solamente un $10 \%$ se dedica únicamente a la docencia (Figura 10). 


\section{Figura 9}

\section{Tipo de Unidad de Información donde laboran los profesores asignatura}

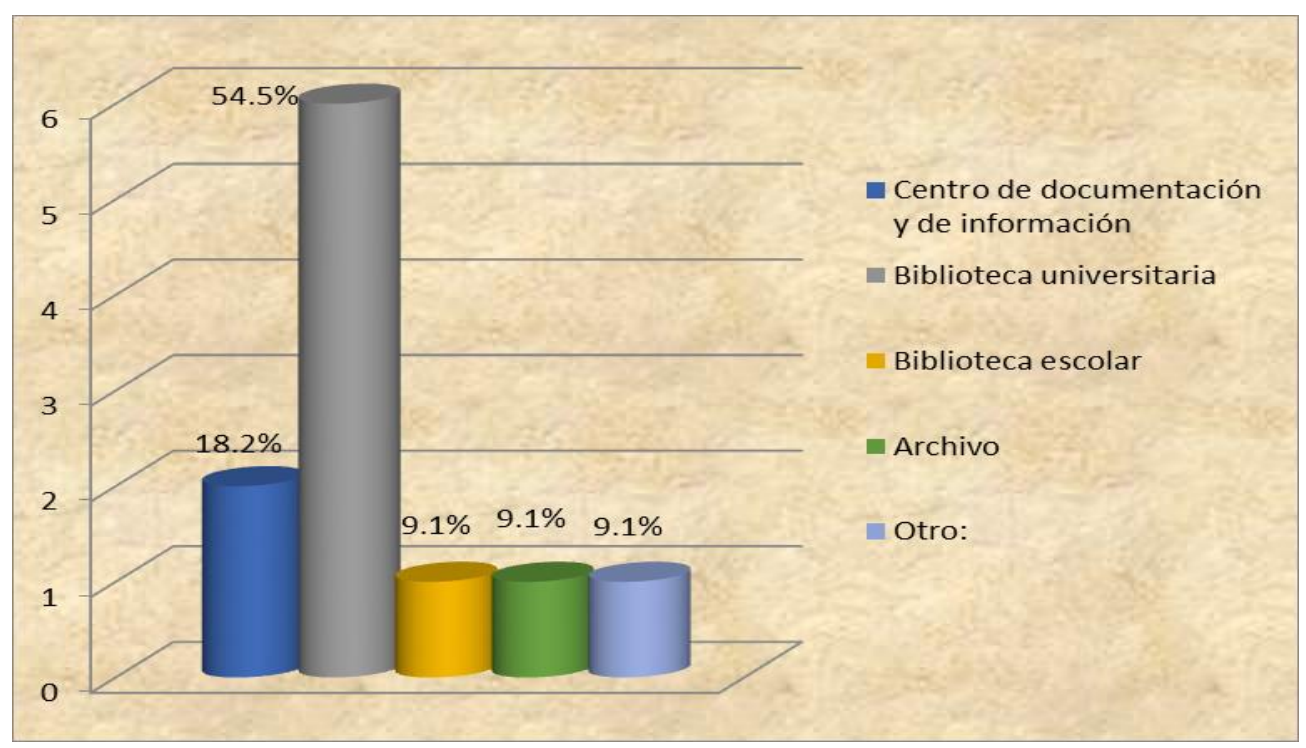

Fuente: Elaboración propia de los autores.

Figura 10

Áreas en que laboran profesionalmente los docentes asignatura

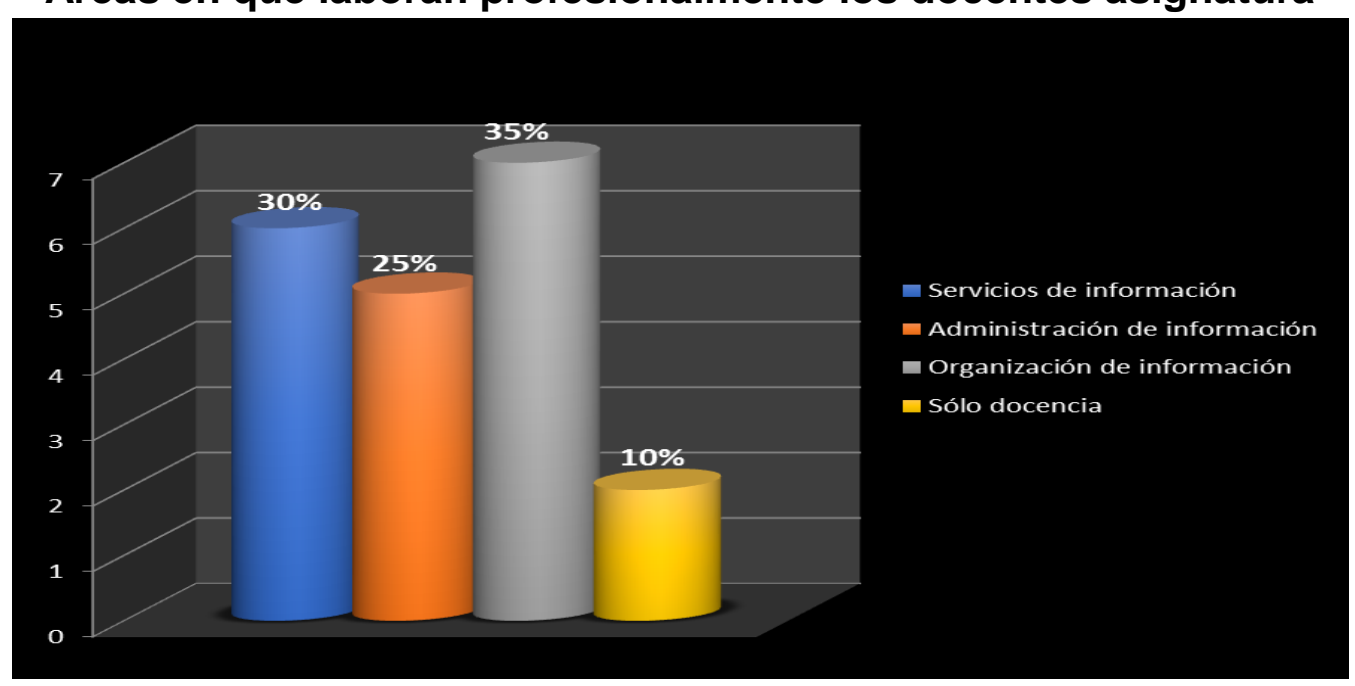

Fuente: Elaboración propia de los autores. 
Por su parte los un $70 \%$ de los docentes manifestó que imparte cursos o talleres fuera de su institución de adscripción; donde solamente un $30 \%$ de ellos afirmó que no imparte cursos o talleres (Figura 11).

Figura 11

Los docentes, ¿imparten cursos o talleres fuera de su institución?

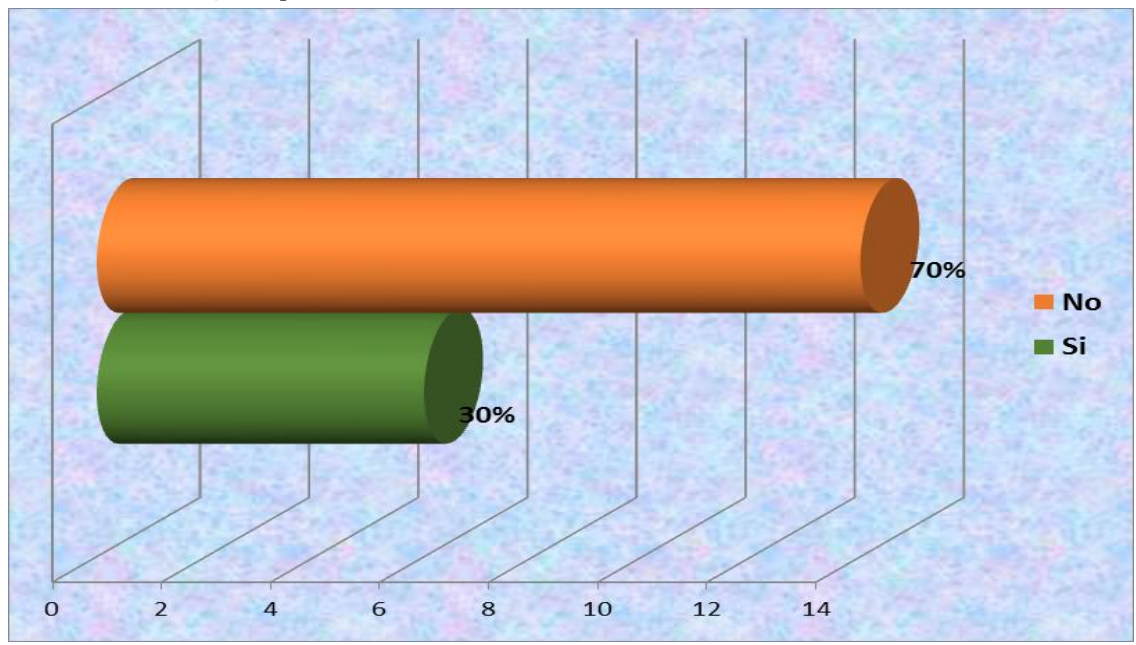

Fuente: Elaboración propia de los autores.

\section{Conclusiones}

Todos los docentes de tiempo completo tiene un compromiso de exclusividad para no trabajar más de ocho horas a la semana fuera de la institución, pero sus actividades hacen que su tiempo laboral sea para la Universidad. Respecto de la antigüedad de los profesores en la institución, esta es muy variable, pues va de uno hasta treinta y tres años. Sobre la temática de los cursos, cada año la Facultad de Ciencias de la Información ofrece cursos a todos los profesores, relacionados con la actividad docente y con las diversas áreas de la gestión de información.

Además, apoya a los profesores para que asistan a eventos nacionales o internacionales. La mayor parte de los profesores asignatura cuentan con un área ejercicio de laboral en la 
organización y difusión de la información, por lo que llevan al aula las situaciones y problemas de las unidades de información. Los profesores que cuentan solo con licenciatura tienen la posibilidad de cursar la Maestría en Ciencias de la Información que ofrece la $\mathrm{FCl}$ y todos los profesores pueden actualizarse en su especialidad docente con los cursos de actualización que se ofrecen ahí anualmente.

\section{Bibliografía}

Álvarez, M. (2011). Perfil del docente en el enfoque basado en competencias. Revista Electrónica Educare, Vol. 25 (1).

Bozu, Z. y Canto, P. (2009). El profesorado universitario en la sociedad del conocimiento: competencias profesionales docentes Revista de Formación e Innovación Educativa Universitaria. Vol. 2 (2).

Díaz-Barriga, Á. (2005). El profesor de educación superior frente a las demandas de los nuevos debates educativos. Perfiles educativos, Vol. 27 (108).

Gimeno-Sacristán, J. (1989). El currículum: una reflexión sobre la práctica. Madrid: Morata.

Tunnermann. C. (2008). Modelos educativos y académicos. Nicaragua: Hispamer.

Universidad Autónoma de San Luis Potosí (2014). Facultad de Ciencias de la Información. Plan de Estudios de la carrera de Gestión de Información. San Luis Potosí, UASLP.

\section{Notas de los autores}

* Dra. Rosa María Martínez Rider: Universidad Autónoma de San Luis Potosí, México. Correo electrónico rriderf@uaslp.mx

** Dra. Adriana Mata Puente: Universidad Autónoma de San Luis Potosí, México. Correo electrónico amata@uaslp.mx

*** Dr. Eduardo Oliva Cruz: Universidad Autónoma de San Luis Potosí, México. Correo electrónico eduardo.oliva@uaslp.mx 